\title{
Treatment of Risk Factors to Prevent Stroke
}

\author{
Junya Aoki $\cdot$ Ken Uchino
}

Published online: 29 June 2011

(C) The American Society for Experimental NeuroTherapeutics, Inc. 2011

\begin{abstract}
Much of the decline in stroke incidence and mortality for the past several decades in Western countries has been attributed to better treatment of risk factors. Many epidemiological studies and clinical trials confirmed the importance of managing hypertension. Comparative trials of anti-hypertensive drugs or drug classes have not yielded clear results, but blood pressure variability may play an important role beyond the absolute value of blood pressure. Diabetes therapy remains a conundrum. Although diabetes is clearly a risk factor for ischemic stroke, treatment trials targeting different glycemic goals have not indicated that glucose lowering results in stroke prevention. Trials focused on insulin resistance are ongoing and they may be able to help establish the management of diabetes/impaired glucose tolerance. Evidence for treatment of dyslipidemia has contrasted science to diabetes mellitus. Dyslipidemia has not been strongly or consistently linked to ischemic stroke but the Stroke Prevention by Aggressive Reduction in Cholesterol Levels (SPARCL) trial showed the impact of statin treatment in stroke prevention. The results of clinical trials investigating dabigatran and rivaroxaban clearly indicate alternative strategies to vitamin $\mathrm{K}$ antagonists in stroke prevention for persons with atrial fibrillation. Evidence for stroke prevention
\end{abstract}

Electronic supplementary material The online version of this article (doi:10.1007/s13311-011-0054-0) contains supplementary material, which is available to authorized users.

\section{J. Aoki}

Department of Stroke Medicine, Kawasaki Medical School, Kurashiki, Okayama 701-0192, Japan

e-mail: aojyun@med.kawasaki-m.ac.jp

K. Uchino $(\bowtie)$

Cerebrovascular Center, Cleveland Clinic,

Cleveland, OH 44195, USA

e-mail: uchinok@ccf.org by life style modification, treating metabolic syndrome, sleep disordered breathing, lipoprotein (a), hyperhomocysteinemia, and coagulation disorders are also discussed.

Keywords Stroke prevention - Risk factors · Treatment . Blood pressure $\cdot$ Cerebrovascular disease

\section{Introduction}

When we examine persons with stroke, we often find risk factors. Although one may look for "the cause," or the direct mechanism of stroke, such as atherosclerotic plaque or embolic source, common risk factors contribute a significant extent to the underlying processes that lead to strokes. Treatment of risk factors is essential to prevent the first and subsequent stroke of all types, including ischemic stroke, intracerebral hemorrhage, and subarachnoid hemorrhage $[1,2]$. The reduction in stroke incidence in Western countries for the past several decades has been attributed to better treatment of risk factors [3, 4]. The Framingham study was the first major epidemiological study that identified specific risk factors associated with cardiovascular disease. Specifically, the study demonstrated a correlation between cigarette smoking, hypertension, impaired glucose intolerance, atrial fibrillation, obesity, and the development of cardiovascular disease. Subsequent epidemiological studies, such as the Honolulu Heart study and Northern Manhattan Stroke Study corroborated these findings and added new data regarding alcohol and inflammation. First, we will discuss the new scientific data regarding the traditional established risk factors of hypertension, diabetes mellitus, dyslipidemia, and atrial fibrillation. Next, life-style related risk factors are discussed, namely smoking, alcohol-consumption, better diet, exer- 
cise, and obesity. Finally, we will discuss ongoing research and controversy regarding less established risk factors of metabolic syndrome, sleep disordered breathing, lipoprotein (a), hyperhomocysteinemia, and coagulation disorders.

\section{Established Risk Factors}

\section{Hypertension}

The goal of antihypertensive treatments is to reduce the incidence of stroke, as well as coronary heart disease (CHD) and renal failure. Meta-analyses of treatment trials have confirmed the impact of blood pressure (BP) observed in previously in epidemiological studies. Lowering of systolic BP by $10 \mathrm{~mm} \mathrm{Hg}$ reduces the risk of stroke by 30 to $40 \%[5,6]$. Most treatment recommendations have focused on reducing the average or usual blood pressure of repeated measurements over multiple visits over time. Studies during the past decade, however, indicate blood pressure variability as an additional risk factor beyond the absolute value of mean or usual BP. These include measurement-tomeasurement variation at the same clinic encounter, visit-tovisit variation, as well as abnormal circadian patterns of BP [7]. The BP level follows circadian rhythm and typically decreases approximately 10 to $20 \%$ during sleep [8]. A study of ambulatory BP monitoring showed that an abnormal diurnal BP of decline of average BP by $\geq 20 \%$ during sleep ("extreme dipping") or rise in nocturnal BP ("reverse dipper") are associated with new occurrence of silent and clinical cerebral ischemia [9]. Orthostatic hypotension is also associated with higher stroke risk [10]. Secondary analyses of a comparative trial of BP regimen suggests that the differences in visit-to-visit variability among the drug regimen accounts for the differences in cardiovascular events better than mean BP differences alone [11]. Therefore, it is inferred that reduction in $\mathrm{BP}$ variation might improve the prognosis of hypertensive patients and among those who exhibit episodic hypertension. How to intervene on these observed patterns remains a challenge.

The absolute value of goal BP and its importance remains uncertain. On 1 hand, the current American Heart Association and the American Stroke Association (AHA/ASA) guidelines for primary stroke prevention recommended BP reduction based on the Seventh Report of the Joint National Committee on Prevention, Detection, Evaluation, and Treatment of High Blood Pressure (JNC-7) [1, 12]. On the other hand, the AHA/ ASA secondary stroke prevention guideline stated that the absolute target BP level and reduction are uncertain, and should be individualized [2]. In general, the JNC-7 stated the traditional goal has been to avoid hypertension with the usual systolic blood pressure (SBP) $<140 \mathrm{mmHg}$ and diastolic blood pressure $<90 \mathrm{mmHg}$. In patients with diabetes or chronic kidney disease the BP goal is $<130 / 80 \mathrm{mmHg}$. However, this aggressive BP control recommendation is not confirmed by trial evidences. Reappraisal of the European Society of Hypertension guideline states that although this recommendation may be wise, it remains controversial [13]. Although additional benefits are suggested by observational studies, the role of more intensive BP lowering has also not been established. In a recent large-scale clinical trial of persons with type 2 diabetes mellitus, the group with SBP target $<120 \mathrm{~mm} \mathrm{Hg}$, indeed, had lower stroke occurrence by $40 \%$ compared to the group with SBP target $<140$, but this was counterbalanced by higher incidence of hypotension, syncope, bradycardia, and hyperkalemia without any impact on death, myocardial infarction, or need for hemodialysis.

In secondary prevention of stroke, blood pressure lowering is clearly effective and important, and appears to confirm that stroke is reduced by $\sim 40 \%$ by reduction of SBP by $10 \mathrm{mmHg}$ [14]. However, are anti-hypertensives given to persons with stroke who have normal BP helpful in preventing strokes? The normotensive subgroup in the Post-Stroke Antihypertensive Treatment Study seems to suggest this to be as a result [14], and a recent meta-analysis suggest that antihypertensives given to normotensive individuals with prior cardiovascular disease or its risk equivalent (i.e., stroke, $\mathrm{CHD}$, congestive heart failure (CHF), or diabetes mellitus) reduced risk of stroke by $23 \%$ [15]. However, the results should be interpreted with caution because the meta-analysis found heterogeneity among studies, and two other secondary stroke prevention studies after stroke, namely the Perindopril Protection against Recurrent Stroke Study (PROGRESS) and the Prevention Regimen for Effectively Avoiding Second Strokes (PRoFESS) study, did not find significant stroke reduction among normotensive persons [16, 17].

The algorithm for treatment of hypertension initiates with lifestyle modifications. Body weight loss, better diet, limited alcohol intake, exercise, and a combination of these decrease BP not only in patients with hypertension but also in those with pre-hypertension (SBP $120-139 \mathrm{mmHg}$ or diastolic blood pressure $80-89 \mathrm{mmHg}$ ) [18]. Better diet includes sodium reduction and the Dietary Approaches to Stop Hypertension (DASH) diet plan, which is a diet rich in fruits, vegetables, and low fat dairy products with a reduced content of dietary cholesterol, as well as saturated and total fat. Pharmacological treatment should be considered in the next step. In JNC-7, thiazide-type diuretics are recommended as the first choice. Diuretics have been used as a basis in many antihypertensive trials and are less expensively available than other agents. While lowering blood pressure appears to be the most important factor, it is less clear whether a particular drug or drug class is advantageous in lowering stroke risk. JNC-7 recommends choosing a drug that is tailored toward individual needs, such as comorbidities of coronary arteries and diabetes mellitus. Trials 
comparing different drugs have not yielded clear consistent results, partly due to differences in BP achieved. Recent analyses in BP variability suggest nonloop diuretics and calcium channel blockers have lower BP variability and may explain the apparent prior conflicting data regarding differential drug class effect on cardiovascular outcomes [7, 19]. The meta-regression analyses results suggested superiority of a calcium channel blocker in comparison with beta-blockers [6]. Another meta-analysis comparing diuretics to renin-angiotensin inhibitors in secondary stroke prevention concluded greater effect seen in diuretics than renin-angiotensin inhibitors [14]. However, before focusing on the idea that 1 drug class is superior to another, it is important to note that more $50 \%$ of persons with hypertension need more than 1 antihypertensive drug in clinical practice [20]. We should pay attention to reduction of BP itself before focusing on specific drug choice.

\section{Diabetes Mellitus}

Approximately $33 \%$ of persons with ischemic strokes have diabetes mellitus, an established risk factor for stroke [21]. Among persons without diabetes, insulin resistance has also been associated with the first stroke and is prevalent in approximately $50 \%$ of persons with transient ischemic attack (TIA) or ischemic stroke [22, 23]. Yet, it remains uncertain whether treatment of hyperglycemia or a particular glycated hemoglobin target is associated with reduction in ischemic stroke or cardiac events. The Action to Control Cardiovascular Risk in Diabetes (ACCORD) trial compared 10,251 persons with diabetes randomized to intensive treatment group with target $\mathrm{A} 1 \mathrm{C}$ level $<6.0 \%$ and a standard group with target A1C level of 7.0 to $7.9 \%$. The study was halted early, because all-cause mortality was higher in the intensive therapy group without any difference in stroke or cardiovascular outcomes [24]. Similarly, the United Kingdom Prospective Diabetes Study (UKPDS), the Action in Diabetes and Vascular Disease (ADVANCE), and the Veterans Affairs Diabetes trial failed to show the benefit of intensive therapy for stroke prevention [25-27]. The American Diabetes Association states that the general glycated hemoglobin goal of $<7.0 \%$ appears reasonable to prevent macrovascular disease in diabetes patients [28].

There is some evidence for advantage or disadvantage of 1 diabetes therapy in comparison to another in preventing stroke or cardiovascular complications. Rosiglitazone garnered attention when meta-analyses of randomized clinical trials noted an increase in risk in myocardial infarction [29, 30]. Data regarding stroke is more limited. A restrospective cohort study comparing two types of thiazolidinediones using United States (U.S.) Medicare prescription data showed that prescription of rosiglitazone compared to pioglitazone resulted in significant 1.3 -fold increase in stroke, as well as myocardial infarction [31]. In 1 metaanalysis of randomized controlled studies, piogliazone appears to have a favorable cardiovascular results, with lower overall 0.82 -fold lower combined endpoint of death, myocardial infarction, or stroke [32]. The individual stroke and myocardial infarction had similar trends without statistical significance. The Prospective Pioglitazone Clinical Trial in Macrovascular Events (PROactive) adds to the evidence for pioglitazone therapy. In this randomized clinical trial among persons with diabetes and evidence of macrovascular complications, adding pioglitazone or placebo to diabetes regimen showed that pioglitazone reduced both overall cardiovascular events as a secondary endpoint in the study and stroke among those with history of stroke [33].

The combination of diabetes with hypertension doubles the stroke risk and triples the risk of coronary heart disease $[34,35]$. Thus, intensive BP reduction toward $<130 /$ $80 \mathrm{mmHg}$ is described in several existing guidelines. The Action to Control Cardiovascular Risk in Diabetes (ACCORD) trial compared the risk of cardiovascular events between the intensive therapy $(<120 \mathrm{mmHg})$ group and the standard therapy $(<140 \mathrm{mmHg})$ group. Cardiovascular events was not different between the two groups. Stroke was assessed as a pre-specified secondary endpoint. Although the total stroke number was small, the intensive therapy group had lower stroke occurrence than the standard therapy group [36]. In contrast, a secondary analysis of the International Verapamil SR-Trandolapril (INVEST) study of patients with diabetes and coronary artery disease did not show any association of tight BP control (average SBP during study $<130 \mathrm{mmHg}$ ) with improved overall cardiovascular outcome or specifically stroke occurrence compared to usual BP control (average SBP during study $130 \mathrm{mmHg}$ to $<140 \mathrm{mmHg}$ ) [37]. At this point the benefit of the aggressive BP control below $130 \mathrm{~mm} \mathrm{Hg}$ remains uncertain.

\section{Dyslipidemia}

High serum levels of total cholesterol or low-density lipoprotein (LDL) cholesterol have not been related to stroke risk overall in multiple observational epidemiological studies [38, 39]. It seems that cholesterol has the opposite relationship to ischemic stroke (positive relation) and hemorrhagic stroke (inverse relation) [40]. Yet, lipid lowering using 3-hydroxy-3methylglutaryl coenzyme A reductase inhibitors ("statins") in clinical trials have reduced overall stroke risk in both at-risk populations and after ischemic stroke [41-43]. Metaregression analysis of data from more than 160,000 participants in 24 randomized trials showed that statin treatment decreases $21 \%$ of the nonfatal first ischemic stroke per $1.0 \mathrm{mmol} / \mathrm{L}(39 \mathrm{mg} / \mathrm{dL})$ reduction in LDL cholesterol.

For those without known ischemic stroke or TIA, an approach to treat dyslipidemia should be based on the 
guideline of the National Cholesterol Education Program Expert Panel on Detection, Evaluation, and Treatment of High Cholesterol on Adults (NCEP ATP III) [44]. The primary target of dyslipidemia treatment is LDL cholesterol. Based on the presence of CHD risk factors (including cigarette smoking, hypertension, high-density lipoprotein (HDL) cholesterol $<40 \mathrm{mg} / \mathrm{dL}, \mathrm{CHD}$ in a male first-degree relative $<55$ years or in a female first-degree relative $<65$ years, or age $>45$ years for men or $>65$ years for women), the 10-year risk calculation and CHD risk equivalents (diabetes or other forms of atherosclerotic disease, such as peripheral arterial disease, abdominal aortic aneurysm, or symptomatic carotid artery disease) and the goal of LDL levels are defined. The 10-year risk calculation is available at www.nhlbi.nih.gov/guidelines/ cholesterol/index.htm. The goal of persons with 0 to 1 risk factor is $<160 \mathrm{mg} / \mathrm{dL}$ and those with 2 risk factors is $<130 \mathrm{mg} / \mathrm{dL}$ when the 10 -year risk is $<20 \%$. High-risk patients with CHD, a CHD risk equivalent, or 2 risk factors with 10 -year risk of $>20 \%$ are recommend to control LDL levels to $<100 \mathrm{mg} / \mathrm{dL}$.

An alternative risk stratification for primary prevention has arisen from the results of the Justification for the Use of Statins in Primary Prevention: An Intervention Trial Evaluating Rosuvastatin (JUPITER) [45, 46]. The study selected apparently low-risk individuals with LDL cholesterol $<130$, but elevated risks were identified by elevated highly sensitive Creactive protein (hsCRP) $>2 \mathrm{mg} / \mathrm{L}$. In this trial, LDL reduction by $50 \%$ and hsCRP reduction by $37 \%$ through rosuvastatin of $20 \mathrm{mg}$ daily was associated with overall cardiovascular event reduction and $48 \%$ reduction in stroke. The absolute risk reduction, however, was $0.59 \%$ per year for combined cardiovascular endpoint and $0.16 \%$ per year for stroke, which would translate to the number needed to treat of 34 for a 5-year time span to prevent 1 MI, stroke, arterial revascularization, unstable angina, or death, or number needed to treat of 125 during the 5 years to prevent 1 stroke.

In patients with a history of non-cardioembolic stroke or TIA without known CHD, the Stroke Prevention by Aggressive Reduction in Cholesterol Levels (SPARCL) trial showed that atorvastatin $(80 \mathrm{mg})$ compared with a placebo lowers overall stroke recurrence by $16 \%$, regardless of the ischemic stroke subtype at entry [42, 47]. The absolute risk reduction during a 5 -year time span was $2.2 \%$, with a number needed to treat of 45 during the 5 years. A greater relative benefit seems to be derived for those with atherosclerosis. Post-hoc analysis showed that $\geq 50 \%$ LDL cholesterol reductions had $31 \%$ reduction in stroke risk and LDL cholesterol level $<70 \mathrm{mg} / \mathrm{dl}$ was significantly associated with a $28 \%$ reduction in risk of stroke [48]. In an apparent paradox, in a meta-analysis the group of secondary prevention of stroke derived a lower relative benefit than primary prevention of stroke [43]. The SPARCL trial had reduced LDL cholesterol by an average of $1.4 \mathrm{mmol} / \mathrm{L}$, which resulted in just $16 \%$ relative reduction. This apparent paradox is explained by the observations that the primary stroke population in meta-analyses derives mostly from patients at risk for atherosclerotic coronary events and when subset of patients in SPARCL is limited to atherosclerotic stroke or those with carotid stenosis, the risk reduction approximates observed in meta-analyses [43, 47]. Although elevation of liver enzymes levels, renal dysfunction, myalgia, and myositis are known side effects of statins, their favorable risk-benefit profile has been shown in clinical trials and observational studies [41, 49]. These findings led to the current AHA/ASA guideline recommendation for patients with "atherosclerotic" ischemic stroke or TIA, and without known CHD, it is reasonable to target a reduction of at least $50 \%$ in LDL cholesterol or a target LDL cholesterol level of $<70 \mathrm{mg} / \mathrm{dL}$ to obtain maximum benefit [50].

Most epidemiological studies suggested the association between lower levels of total and LDL cholesterol and higher risk of hemorrhagic stroke [51, 52]. In the SPARCL trial, statin increased the incidence of hemorrhagic stroke, but overall stroke risk was reduced. The subgroup that appeared not to benefit from atorvastatin was those with hemorrhagic stroke at entry [47]. No relation between risk of hemorrhage and baseline or recent LDL cholesterol level was observed in patients treated with statin, and only those with hemorrhagic stroke at entry appeared not to benefit from statin treatment [53]. In addition, meta-analyses have not yielded increased risk of hemorrhagic stroke with statins, although the data relies mostly on patients without prior stroke [41, 43]. Caution for statin use is advised for those who has suffered cerebral hemorrhage without known CHD. Statin use for those who have coronary artery disease and spontaneous cerebral hemorrhage remains less certain.

It is not clear whether lipid-lowering drugs other than statins reduce stroke risk. Ezetimibe reduces LDL cholesterol, and a large-scale trial to study its effects on clinical outcome is ongoing, but to date, it has failed to show any effect on measured atherosclerosis biomarkers [54, 55]. Niacin (also known as nicotinic acid, vitamin $\mathrm{B}_{3}$ ) increases HDL cholesterol and reduces LDL cholesterol, but has not been studied in well-designed trials with clinical outcomes. In a recent study comparing ezetimibe to niacin given in conjunction with a statin demonstrated reduction by niacin in atherosclerosis (measured by intima-media thickness) and cardiovascular events (stroke was not reported and was probably too infrequent to generate interpretable results) [55]. Although triglycerides are not consistently associated with stroke in epidemiologic studies, it has been a target in clinical trials of cardiovascular prevention using fibrates, such as gemfibrozil and fenofibrate. These drugs reduce both LDL cholesterol and triglycerides and raise HDL slightly. Gemfibrozil reduced myocardial infarction and 
stroke among men with coronary artery disease with low HDL and LDL in the Veterans Affairs HDL Intervention Trial (VA-HIT), but recent meta-analysis of fibrates suggested that it has effects on coronary events, but no effect on stroke [56, 57].

\section{Atrial Fibrillation}

Anticoagulation is available for stroke prevention in patients with atrial fibrillation. As primary stroke prevention, anticoagulation is recommended to all patients with atrial fibrillation at high or moderate risk for stroke who can receive it safely [1]. For patients with ischemic stroke or TIA with atrial fibrillation, anticoagulation is recommended as an established prevention strategy [2]. Warfarin adjusted to international normalized ratio between 2 and 3 reduces stroke incidence by $64 \%$ compared to a placebo [58]. Warfarin compared to aspirin provides further reduction of stroke by 39\% [59]. The expected baseline stroke risk and the risk of bleeding should help in the decision to choose warfarin or aspirin. The CHADS2 scoring system has been developed to select moderate-to-high-risk persons with atrial fibrillation that would benefit from anticoagulation therapy. The " $\mathrm{C}$ " indicates the presence of congestive heart failure; the " $\mathrm{H}$ " indicates hypertension; the " $\mathrm{A}$ " stands for age $\geq 75$; the " $\mathrm{D}$ " is for diabetes with a score of 1 point; the "S" represents a history of stroke or TIA with a score of 2 points. Patients with atrial fibrillation and a CHADS2 score of $\geq 2$ are defined as high risk of stroke (1.9\% to $7.6 \%$ annually) and are recommended to receive warfarin [60]. To select more candidates among patients with CHADS2 score ranging from 0 to 1 , the European Society of Cardiology suggested the use of CHA2DS2-VASc scoring system [61].

Direct thrombin inhibitors that do not require dose adjustment or monitoring are being developed. Dabigatran does not require dose adjustment and has been shown to be a good alternative to warfarin in preventing stroke in an openlabel randomized clinical trial [62]. The U.S. Food and Drug Administration approved dabigatran for the indication of stroke prevention in atrial fibrillation in October 2010. The dose of $150 \mathrm{mg}$ twice daily appears to prevent stroke better than warfarin without higher hemorrhagic complications rates, except in gastrointestinal bleeding. A direct factor Xa inhibitor rivaroxaban is also expected to be an alternative to oral anticoagulant and does not need routine blood coagulation analysis. In a recently completed comparative trial, rivaroxaban taken once daily has been demonstrated not to be inferior to adjusted warfarin in prevention of stroke and other embolism with similar rates of hemorrhagic complications and lower rates of intracranial hemorrhage [63].

For those who cannot use anticoagulation, use of antiplatelet therapy for patients with atrial fibrillation has been studied. Aspirin alone reduces strokes in atrial fibrillation by $\sim 22 \%$ [58]. The Atrial Fibrillation Clopidogrel Trial with Irberartan for Prevention of Vascular Events (ACTIVE)-A \& W studies failed to show the clinical benefit of combined antithrombotic therapy of aspirin and clopidogrel [64, 65]. In these trials, an adverse effect of hemorrhage was significantly higher in aspirin-clopidogrel combination therapy compared to aspirin or warfarin. When patients with atrial fibrillation need temporary interruption of oral anticoagulation, a lowmolecular-weight heparin bridge is reasonable [50].

Nonpharmacologic approaches in preventing stroke in atrial fibrillation include attempts to stop the rhythm of atrial fibrillation and to occlude the left atrial appendage, in which most thrombus in atrial fibrillation is believed to arise. In selected patients, the Cox maze procedures achieve $>80 \%$ freedom from atrial fibrillation at 1-year follow-up [66]. Catheter-based ablation of atrial fibrillation is less invasive and seems to reduce recurrence of atrial fibrillation in selected patients by $\sim 70 \%[67,68]$. However, these attempts to eliminate the rhythm still have significant atrial fibrillation recurrence at approximately $25 \%$ in controlled trials and should not be an approach to avoid anticoagulation. Devices to occlude left atrial appendage have demonstrated relative safety and might reduce stroke risk without anticoagulation. However, the clinical trial with Percutaneous Left Atrial Appendage Transcatheter Occlusion (PLAATO) device was an uncontrolled trial and only compared to the expected rate of stroke occurrence [69]. The randomized trial that compared the WATCHMAN device (Atritech, Incorporated, Plymouth, Minnesota) with warfarin recruited persons who had atrial fibrillation with relative low stroke risk, resulting in low event rates [70]. The study protocol required 45 days of warfarin after device implantation, limiting applicability to those who cannot tolerate anticoagulation even in the short-term. In addition, significant procedure-related complication was reported especially early in the trial. These procedures may overcome limitations of medical treatment and warrant proper scientific investigation.

\section{Lifestyle Changes}

\section{Smoking}

Cigarette smoking or passive smoking is one of the modifiable risk factors [71]. Cigarette smoking causes endothelial dysfunction, hypercoagulability, and inflammation, resulting in progression of atherosclerosis. Although the risk of smokeless tobacco is relatively low, smokeless tobacco is also associated with an increased risk of fatal stroke [72]. Current smokers have a 2-fold risk of ischemic stroke and subarachnoid hemorrhage. A dose-response relationship exists between cigarette smoking and stroke risk. Smoking is a modifiable risk factor and cessation of 
smoking reduces the risk of stroke and other outcomes after several years of quitting [73]. In a cohort study of women, the stroke and cardiovascular mortality reached a comparable risk to never smokers after 20 years of cessation [74]. Combination of pharmacotherapy interventions and behavioral counseling are available to stop smoking. The U.S. Food and Drug Administration has approved 7 types of smoking cessation agents. Five are nicotine replacement products (gum, inhaler, lozenge, patch, and nasal spray) and 2 are non-nicotine pharmacologic agents (bupropion sustained-release and varenicline). Clonidine and nortriptyline should only be used in patients who do not tolerate the approved agents due to potential adverse effects. The role of smokeless tobacco as an alternative method of smoking cessation remains uncertain and controversial, because it still may confer health risk and is not consistently associated with smoking cessation.

\section{Alcohol Consumption}

Avoidance of heavy consumption of alcohol is important to reduce stroke occurrence. On one hand, a dose-response relationship has been observed between hemorrhagic stroke (intracerebral hemorrhage and subarachnoid hemorrhage) and the amount of alcohol consumed. On the other hand, a "J"shaped association between ischemic stroke and alcohol consumption has been reported [75]. Mild alcohol intake decreases fasting insulin, improves insulin sensitivity and lipid profile, and increases the adiponection plasma level, but the true mechanism of detrimental and beneficial effects of alcohol remains unknown [76]. Recent meta-analysis showed that in male alcohol consumption of less than $35 \mathrm{~g} /$ day, or less than 3 drinks per day based on U.S. conversions, was significantly associated with a decreased relative risk for ischemic stroke, and the risk curve had a global minimum for $12 \mathrm{~g}$ of pure alcohol or 1 drink per day. For women, the lowest risk of mortality of ischemic stroke was among those consuming less than $12 \mathrm{~g} /$ day, or about 1 drink/day [77]. The ASA/AHA guideline stated that continuation of light-to-moderate alcohol consumption may be reasonable [2]. Yet, it is important to note that these epidemiological observational studies cannot completely overcome the possibilities of confounding (i.e., healthy cohort effect) and there are other negative health consequences of alcohol (e.g., trauma, liver disease). Current AHA guidelines ended with the following sentences: "nondrinkers should not be counseled to start drinking" [2].

\section{Dietary Habits}

Weight control, reduced intake of sodium, and increased dietary potassium prevent and treat hypertension [78]. In epidemiological studies, vegetarians are more likely to have lower blood pressures than nonvegetarians [79]. One of the reasons may be that they consume more, potassium, and magnesium. The effect of blood pressure lowering has also been shown by sodium reduction and the Dietary Approaches to Stop Hypertension (DASH) diet, which is low in saturated and total fats, but rich in fruits, vegetables, and low-fat dairy products [80]. In addition, the DASH diet likely reduces the components of metabolic syndrome. The DASH diet also provides a favorable effect on blood lipid profile and other components of the metabolic syndrome, with lower total, LDL, and higher HDL cholesterol concentrations and improved insulin sensitivity. Observational data indicated that increased fruit and vegetable intake in the range commonly consumed and dietary habits conforming to the DASH diet are associated with a reduced risk of stroke [81, 82].

The Mediterranean diet has also gained attention. It also seems to have beneficial effects in reducing myocardial infarction, but the effects on stroke is less clear [83, 84]. Among older adults, modest consumption of fish (not fried fish) was associated with lower prevalence of subclinical infarcts and white matter abnormalities on magnetic resonance images [85]. These data support the benefit of lifestyle modification.

\section{Physical Inactivity}

In epidemiological studies comparing highly active to sedentary individuals, physical activity are observed to reduce the risk of both ischemic and hemorrhagic strokes [86, 87]. The 2008 Physical Activity Guidelines for Americans concur in recommending regular physical activity to reduce adverse health outcomes [88]. In this guideline, most health benefits occur with at least 150 minutes a week of moderate intensity, or 75 minutes a week of vigorous intensity of aerobic physical activity. When adults with disabilities are not able to meet these guidelines, they should engage in regular physical activity according to their abilities, and they should avoid inactivity. Exercise reduces blood pressure, decreases body weight, and improves other risk factors, including metabolic syndrome [89]. The role of the health provider is important and the physician's advice regarding physical activity to patients can significantly modify exercise and diet style, including those with a prior stroke [90]. One recent observation of note is associated with the onset of stroke with physical activity [91]. There might be an increased risk of stroke occurrence within 1 hour of moderate or vigorous physical activity.

Obesity

Obesity is commonly defined using body mass index (BMI), a measurement of body weight; body weight $(\mathrm{kg})$ divided by the square of his or her height $\left(\mathrm{m}^{2}\right)$. BMI between 18.5 and 24.9 $\left(\mathrm{kg} / \mathrm{m}^{2}\right)$ is categorized as normal, BMI between 25.0 and 
$30\left(\mathrm{~kg} / \mathrm{m}^{2}\right)$ is categorized as overweight, and BMI greater than $30\left(\mathrm{~kg} / \mathrm{m}^{2}\right)$ is obese. Epidemiological data indicate that the risk of stroke-related mortality increases linearly when greater than a BMI of $25 \mathrm{~kg} / \mathrm{m}^{2}$ (i.e., greater than normal range [92]). This finding is likely due to increased stroke incidence with increasing weight and not increased mortality after stroke [93], because paradoxically, obesity may be associated with reduced long-term mortality after stroke. Some studies have demonstrated a protective effect of increasing BMI or obesity category in both the ischemic stroke and intracerebral hemorrhage [94, 95]. The phenomenon may be limited to the elderly individuals [96]. Although there is no randomized trial that investigated the benefit of weight reduction for the purpose of reducing stroke incidence, many studies show control of weight is associated with BP and insulin sensitivity, and thereby may reduce the risk of stroke [97].

\section{Putative Risk Factors under Investigation}

\section{Metabolic Syndrome}

The metabolic syndrome is a constellation of physical and metabolic characteristics that are associated with higher cardiovascular risk, including abdominal obesity, insulin resistance, hypertriglyceridemia, low levels of high-density lipoprotein cholesterol, and hypertension. The National Cholesterol Education Program criteria for the metabolic syndrome are to have 3 of the 5 risk factors. Many trials have reported the increased risk of total and ischemic strokes independently of other known factors, such as alcohol consumption, LDL cholesterol, and smoking [98]. However, in assessing and treating cardiovascular risk, it is not clear whether the concept and definition of the metabolic syndrome adds beyond the individual risk factors that compose the metabolic syndrome. For patients and physicians, the concept of the syndrome might be a useful tool in recognizing the frequent combination of the risk factors. The management of metabolic syndrome is not clearly established and individual components should be treated, including lifestyle modification with diet and exercise to decrease the abdominal obesity.

\section{Sleep Disordered Breathing}

Sleep disordered breathing (SDB) is observed in the majority of stroke patients [99]. Obstructive sleep apnea syndrome (OSA) is the most common form of SDB affecting 5 to $15 \%$ of the general population. Cheyne-Stokes respiration and central sleep apnea are other types of SDB. The main treatment for OSA syndrome is the use continuous positive airway pressure (CPAP), which can provide mild reductions in blood pressure, an effect that might decrease stroke risk
[100]. Oxygen, mechanical ventilation, and tracheostomy are available options in patients with central sleep apnea, central hypoventilation, and Cheyne-Stroke respiration. Although CPAP compliance was $70 \%$ in the rehabilitation setting, in the acute phase the rate remains at approximately $25 \%$ [101]. In another study, stroke patients with SDB who did not tolerate CPAP had higher 5-year mortality than those who tolerated CPAP [102]. Beyond hypertension and obesity, the link between SDB and stroke may include intermittent hypoxemia of OSA and pulmonary vasoconstriction, increased pulmonary artery, pressure, diastolic cardiac dysfunction, and atrial fibrillation. Patients with untreated OSA have a higher recurrence of $\mathrm{AF}$ after cardioversion and treating OSA may reduce the risk of arrhythmia [103]. In this theory, patients with OSA may benefit from screening for $\mathrm{AF}$, because both OSA and AF predispose to stroke.

\section{Lipoprotein(a)}

Lipoprotein(a) is similar to low-density lipoprotein and is associated with atherogenesis and coronary heart disease [104]. Independent relationship of lipoprotein(a) with stroke and carotid atherosclerosis are also reported $[105,106]$. When LDL goals are achieved, lowering lipoprotein(a) level or raising HDL level can be considered as targets of intervention. However, the benefit of reduction in lipoprotein(a) is not established. Niacin lowers lipoprotein(a), but it also lowers LDL cholesterol and triglycerides and raises HDL cholesterol levels [107, 108]. With these favorable changes in multiple lipid measures, niacin supplementation seems to reduce atherosclerosis and have a protective effect against stroke in a meta-analysis [55, 109]. However, more trial evidence is needed to determine whether lowering lipoprotein(a) actually works in the previously mentioned theory and reduces vascular events, especially stroke occurrence.

\section{Hyperhomocysteinemia}

Higher levels of homocysteine (a metabolite of methionine) have been associated with venous thrombosis, atherogenesis, myocardial infarction, and stroke [110, 111]. Homocysteine levels is influenced by diet, B vitamins, and folic acid, renal function, and genetic composition, including allele variants of $\beta$-cystathione synthase and methylenetetra-hydro-folate reductase. The mechanism by which homocysteine increases stroke risks is unclear. Homocysteine increases thrombotic risk, as evidenced by its association with venous thromboembolism [111, 112]. It has been less consistently linked with markers of atherosclerosis [113]. Hyperhomocysteinemia can be treated by B vitamin supplementation. Folate and vitamin B12 combination can decrease homocysteine levels than folate alone [114]. However, it is uncertain whether reduction of 
homocysteine level is accompanied by reduction of stroke. Whereas individual trials of folic acid or combination $\mathrm{B}$ vitamin supplementation have failed to demonstrate reduction in cardiovascular endpoints, meta-analyses have reported modest, if any, lowering of stroke risk. This finding in meta-analysis is greater with combination supplementation after therapy duration of several years and in populations without folic acid fortification of grain [115]. In addition, because the large scale clinical trials of folic acid and vitamin supplementation did not screen for hyperhomocysteinemia for the trial enrollment, it remains even more unclear whether homocysteine testing is worthwhile.

\section{Thrombophilia}

Most coagulation disorders are associated with venous thrombosis and are weakly, if any, associated with arterial ischemic stroke. The role of hereditary thrombophilia, such as factor V Leiden, prothrombin 20210 mutation, proteins $\mathrm{C}$ and $\mathrm{S}$, and anti-thrombin deficiency, as risk factors of arterial ischemic stroke is not clear. Multiple large casecontrol studies have not convincingly shown the association of the inherited thrombophilias with ischemic stroke, even in young patients $>18$ years old or patients with patent foramen ovale [116]. These venous thrombotic factors may still be relevant as a factor in stroke-selected patients within the young [117]. In addition, greater relevance for secondary prevention is the question whether there is a higher risk of stroke recurrence in this population. Limited data of small studies do not indicate greatly increased risk [118]. Among children, a meta-analysis indicates that thrombophilia has a stronger role in pediatric arterial ischemic stroke and cerebral venous thrombosis [119]. Due to this uncertain association, a person with arterial ischemic stroke with venous thrombophilia does not require anticoagulation, but it can be considered.

The presence of anti-phospholipid antibodies, including anti-cardiolipin antibodies (immunoglobulin $\mathrm{M}$ and immunoglobulin G types), lupus anticoagulant, or anti- $\beta_{2}$ glycoproteinI antibody ( $\beta_{2}$ GPI) are all considered acquired autoimmune thrombophilia. The consensus criteria for anti-phospholipid syndrome require thrombotic event and persistent elevated levels of anti-phospholipids [120]. Stroke patients can easily qualify if laboratory abnormalities are persistent. Data from various populations (population-based study or hospital based study; all ischemic strokes, selected by age or selected for lack of causative stroke mechanism) and study design (cohort vs case control) are conflicting in regard to the importance of the laboratory markers. A case control study has reported elevation of anti-cardiolipin among $9.7 \%$ of ischemic stroke patients compared to $4.3 \%$ among control subjects, indicating greater than 2-fold odds of anti-cardiolipin antibody elevation among ischemic stroke persons [121]. Larger case-cohort studies have not consistently indicated higher prevalence of anti-cardiolipin antibodies among stroke compared to controls $[122,123]$. In another study, anti-cardiolipin antibodies were detectable in $11 \%$ of 1020 elderly controls without any clinical consequences [124]. Higher titers of anti-cardiolipin antibodies might not be associated with higher stroke recurrence risk [125]. The Antiphospholipid Antibody and Stroke Study (a substudy of a randomized clinical trial) demonstrated no association of the presence of anticardiolipin antibodies with either subsequent vascular occlusive events or a differential response to aspirin or warfarin therapy among general ischemic stroke patients [126]. Compared to less specific anti-cardiolipin antibodies, lupus anticoagulant may pose a stronger risk, particular among young women [127]. Thus, anti-platelet therapy can be considered for primary stroke prevention in persons with anti-phospholipid antibodies alone, particularly of lupus anticoagulant and data casts doubt on anticoagulation. Although some advocate warfarin anticoagulation at high intensity with international normalized rate target $>3$ for those meeting the criteria for anti-phospholipid syndrome, the studies on stroke have not established anticoagulation at any intensity with any certainty [128]. Oral anticoagulation may be helpful in selected population.

\section{Conclusion}

Management of risk factors is critical to prevent stroke. Not only pharmacological treatment, but also life-style modification including diet and exercise are recommended. Evidence-based recommendations found in treatment guidelines are useful, especially for hypertension, dyslipidemia, and atrial fibrillation in which the clinical trial evidence is rich. Yet there are many established and lessestablished risk factors lacking scientific evidence for screening and treatment. Although science seeks independent contribution of each risk factor to disease, management to one can bring additional improvement of other factors in practice and the accumulation of each improvement will finally decrease the stroke risk. Further investigations are much needed to establish the best management of important and common risk factors.

Acknowledgment Full conflict of interest disclosure is available in the electronic supplementary material for this article.

\section{References}

1. Goldstein LB, Bushnell CD, Adams RJ, et al. Guidelines for the Primary Prevention of Stroke: A Guideline for Healthcare Professionals from the American Heart Association/American Stroke Association. Stroke 2011;42:517-584. 
2. Furie KL, Kasner SE, Adams RJ, et al. Guidelines for the prevention of stroke in patients with stroke or transient ischemic attack: a guideline for healthcare professionals from the American Heart Association/American Stroke Association. Stroke 2011;42:227-276.

3. Rothwell PM, Coull AJ, Giles MF, et al. Change in stroke incidence, mortality, case-fatality, severity, and risk factors in Oxfordshire, UK from 1981 to 2004 (Oxford Vascular Study). Lancet 2004;363:1925-1933.

4. Islam MS, Anderson CS, Hankey GJ, et al. Trends in incidence and outcome of stroke in Perth, Western Australia during 1989 to 2001: the Perth Community Stroke Study. Stroke 2008;39:776782.

5. Neal B, MacMahon S, Chapman N. Effects of ACE inhibitors, calcium antagonists, and other blood-pressure-lowering drugs: results of prospectively designed overviews of randomised trials. Blood Pressure Lowering Treatment Trialists' Collaboration. Lancet 2000;356:1955-1964.

6. Lawes CM, Bennett DA, Feigin VL, Rodgers A. Blood pressure and stroke: an overview of published reviews. Stroke 2004;35:1024.

7. Rothwell PM. Limitations of the usual blood-pressure hypothesis and importance of variability, instability, and episodic hypertension. Lancet 2010;375:938-948.

8. Pickering TG. Blood pressure variability and ambulatory monitoring. Curr Opin Nephrol Hypertens 1993;2:380-385.

9. Kario K, Pickering TG, Matsuo T, Hoshide S, Schwartz JE, Shimada K. Stroke prognosis and abnormal nocturnal blood pressure falls in older hypertensives. Hypertension 2001;38:852-857.

10. Fagard RH, De Cort P. Orthostatic hypotension is a more robust predictor of cardiovascular events than nighttime reverse dipping in elderly. Hypertension 2010;56:56-61.

11. Rothwell PM, Howard SC, Dolan E, et al. Effects of beta blockers and calcium-channel blockers on within-individual variability in blood pressure and risk of stroke. Lancet Neurol 2010;9:469-480.

12. Chobanian AV, Bakris GL, Black HR, et al. Seventh report of the Joint National Committee on Prevention, Detection, Evaluation, and Treatment of High Blood Pressure. Hypertension 2003;42:12061252.

13. Mancia G, Laurent S, Agabiti-Rosei E, et al. Reappraisal of European guidelines on hypertension management: a European Society of Hypertension Task Force document. J Hypertens 2009;27:2121-2158.

14. Liu L, Wang Z, Gong L, et al. Blood pressure reduction for the secondary prevention of stroke: a Chinese trial and a systematic review of the literature. Hypertens Res 2009;32:1032-1040.

15. Thompson AM, Hu T, Eshelbrenner CL, Reynolds K, He J, Bazzano LA. Antihypertensive treatment and secondary prevention of cardiovascular disease events among persons without hypertension: a meta-analysis. JAMA 2011;305:913-922.

16. PROGRESS Collaborative Group. Randomised trial of a perindopril-based blood-pressure-lowering regimen among 6,105 individuals with previous stroke or transient ischaemic attack. Lancet 2001;358:1033-1041.

17. Yusuf S, Diener HC, Sacco RL, et al. Telmisartan to prevent recurrent stroke and cardiovascular events. $\mathrm{N}$ Engl $\mathrm{J}$ Med 2008;359:1225-1237.

18. Major outcomes in high-risk hypertensive patients randomized to angiotensin-converting enzyme inhibitor or calcium channel blocker vs diuretic: The Antihypertensive and Lipid-Lowering Treatment to Prevent Heart Attack Trial (ALLHAT). JAMA 2002;288:2981-2997.

19. Webb AJ, Fischer U, Mehta Z, Rothwell PM. Effects of antihypertensive-drug class on interindividual variation in blood pressure and risk of stroke: a systematic review and metaanalysis. Lancet 2010;375:906-915.
20. Cushman WC, Ford CE, Cutler JA, et al. Success and predictors of blood pressure control in diverse North American settings: the antihypertensive and lipid-lowering treatment to prevent heart attack trial (ALLHAT). J Clin Hypertens (Greenwich) 2002;4:393-404.

21. Woo D, Gebel J, Miller R, et al. Incidence rates of first-ever ischemic stroke subtypes among blacks: a population-based study. Stroke 1999;30:2517-2522.

22. Rundek T, Gardener $\mathrm{H}, \mathrm{Xu} \mathrm{Q}$, et al. Insulin resistance and risk of ischemic stroke among nondiabetic individuals from the northern Manhattan study. Arch Neurol 2010;67:1195-1200.

23. Kernan WN, Inzucchi SE, Viscoli CM, et al. Impaired insulin sensitivity among nondiabetic patients with a recent TIA or ischemic stroke. Neurology 2003;60:1447-1451.

24. Gerstein HC, Miller ME, Byington RP, et al. Effects of intensive glucose lowering in type 2 diabetes. $N$ Engl J Med 2008;358:2545-2559.

25. Effect of intensive blood-glucose control with metformin on complications in overweight patients with type 2 diabetes (UKPDS 34). UK Prospective Diabetes Study (UKPDS) Group. Lancet 1998;352:854-865.

26. Patel A, MacMahon S, Chalmers J, et al. Intensive blood glucose control and vascular outcomes in patients with type 2 diabetes. $\mathrm{N}$ Engl J Med 2008;358:2560-2572.

27. Duckworth W, Abraira C, Moritz T, et al. Glucose control and vascular complications in veterans with type 2 diabetes. $\mathrm{N}$ Engl $\mathrm{J}$ Med 2009;360:129-139.

28. Skyler JS, Bergenstal R, Bonow RO, et al. Intensive glycemic control and the prevention of cardiovascular events: implications of the ACCORD, ADVANCE, and VA diabetes trials: a position statement of the American Diabetes Association and a scientific statement of the American College of Cardiology Foundation and the American Heart Association. Circulation 2009;119:351357.

29. Nissen SE, Wolski K. Effect of rosiglitazone on the risk of myocardial infarction and death from cardiovascular causes. N Engl J Med 2007;356:2457-2471.

30. Singh S, Loke YK, Furberg CD. Long-term risk of cardiovascular events with rosiglitazone: a meta-analysis. JAMA 2007;298:1189-1195.

31. Graham DJ, Ouellet-Hellstrom R, MaCurdy TE, et al. Risk of acute myocardial infarction, stroke, heart failure, and death in elderly Medicare patients treated with rosiglitazone or pioglitazone. JAMA 2010;304:411-418.

32. Lincoff AM, Wolski K, Nicholls SJ, Nissen SE. Pioglitazone and risk of cardiovascular events in patients with type 2 diabetes mellitus: a meta-analysis of randomized trials. JAMA 2007;298:1180-1188.

33. Wilcox R, Bousser MG, Betteridge DJ, et al. Effects of pioglitazone in patients with type 2 diabetes with or without previous stroke: results from PROactive (PROspective pioglitAzone Clinical Trial In macroVascular Events 04). Stroke 2007;38:865-873.

34. Davis TM, Millns H, Stratton IM, Holman RR, Turner RC. Risk factors for stroke in type 2 diabetes mellitus: United Kingdom Prospective Diabetes Study (UKPDS) 29. Arch Intern Med 1999;159:1097-1103.

35. Stamler J, Vaccaro O, Neaton JD, Wentworth D. Diabetes, other risk factors, and 12-yr cardiovascular mortality for men screened in the Multiple Risk Factor Intervention Trial. Diabetes Care 1993;16:434-444.

36. Cushman WC, Evans GW, Byington RP, et al. Effects of intensive blood-pressure control in type 2 diabetes mellitus. $\mathrm{N}$ Engl J Med 2010;362:1575-1585.

37. Cooper-DeHoff RM, Gong Y, Handberg EM, et al. Tight blood pressure control and cardiovascular outcomes among hyperten- 
sive patients with diabetes and coronary artery disease. JAMA 2010;304:61-68.

38. Psaty BM, Anderson M, Kronmal RA, et al. The association between lipid levels and the risks of incident myocardial infarction, stroke, and total mortality: The Cardiovascular Health Study. J Am Geriatr Soc 2004;52:1639-1647.

39. Willey JZ, Xu Q, Boden-Albala B, et al. Lipid profile components and risk of ischemic stroke: the Northern Manhattan Study (NOMAS). Arch Neurol 2009;66:1400-1406.

40. Tirschwell DL, Smith NL, Heckbert SR, et al. Association of cholesterol with stroke risk varies in stroke subtypes and patient subgroups. Neurology 2004;63:1868-1875.

41. Baigent C, Blackwell L, Emberson J, et al. Efficacy and safety of more intensive lowering of LDL cholesterol: a meta-analysis of data from 170,000 participants in 26 randomised trials. Lancet 2010;376:1670-1681

42. Amarenco P, Bogousslavsky J, Callahan A 3rd, et al. High-dose atorvastatin after stroke or transient ischemic attack. N Engl J Med 2006;355:549-559.

43. Amarenco P, Labreuche J. Lipid management in the prevention of stroke: review and updated meta-analysis of statins for stroke prevention. Lancet Neurol 2009;8:453-463.

44. Grundy SM, Cleeman JI, Merz CN, et al. Implications of recent clinical trials for the National Cholesterol Education Program Adult Treatment Panel III guidelines. Circulation 2004;110:227-239.

45. Ridker PM, Danielson E, Fonseca FA, et al. Rosuvastatin to prevent vascular events in men and women with elevated Creactive protein. N Engl J Med 2008;359:2195-2207.

46. Everett BM, Glynn RJ, MacFadyen JG, Ridker PM. Rosuvastatin in the prevention of stroke among men and women with elevated levels of C-reactive protein: Justification for the Use of Statins in Prevention: an Intervention Trial Evaluating Rosuvastatin (JUPITER). Circulation 2010;121:143-150.

47. Amarenco P, Benavente O, Goldstein LB, et al. Results of the Stroke Prevention by Aggressive Reduction in Cholesterol Levels (SPARCL) trial by stroke subtypes. Stroke 2009;40:1405-1409.

48. Amarenco P, Goldstein LB, Szarek M, et al. Effects of intense low-density lipoprotein cholesterol reduction in patients with stroke or transient ischemic attack: the Stroke Prevention by Aggressive Reduction in Cholesterol Levels (SPARCL) trial. Stroke 2007;38:3198-3204.

49. Athyros VG, Tziomalos K, Gossios TD, et al. Safety and efficacy of long-term statin treatment for cardiovascular events in patients with coronary heart disease and abnormal liver tests in the Greek Atorvastatin and Coronary Heart Disease Evaluation (GREACE) Study: a post-hoc analysis. Lancet 2010;376:19161922.

50. Furie KL, Kasner SE, Adams RJ, et al. Guidelines for the Prevention of Stroke in Patients With Stroke or Transient Ischemic Attack. A Guideline for Healthcare Professionals From the American Heart Association/American Stroke Association. Stroke 2010

51. Noda H, Iso H, Irie F, et al. Low-density lipoprotein cholesterol concentrations and death due to intraparenchymal hemorrhage: the Ibaraki Prefectural Health Study. Circulation 2009;119:21362145.

52. Iso H, Jacobs DR, Jr., Wentworth D, Neaton JD, Cohen JD. Serum cholesterol levels and six-year mortality from stroke in 350,977 men screened for the multiple risk factor intervention trial. N Engl J Med 1989;320:904-910.

53. Goldstein LB, Amarenco P, Szarek M, et al. Hemorrhagic stroke in the Stroke Prevention by Aggressive Reduction in Cholesterol Levels study. Neurology 2008;70:2364-2370.

54. Kastelein JJ, Akdim F, Stroes ES, et al. Simvastatin with or without ezetimibe in familial hypercholesterolemia. $\mathrm{N}$ Engl $\mathrm{J}$ Med 2008;358:1431-1443.
55. Taylor AJ, Villines TC, Stanek EJ, et al. Extended-release niacin or ezetimibe and carotid intima-media thickness. N Engl J Med 2009;361:2113-2122.

56. Bloomfield Rubins H, Davenport J, Babikian V, et al. Reduction in stroke with gemfibrozil in men with coronary heart disease and low HDL cholesterol: The Veterans Affairs HDL Intervention Trial (VA-HIT). Circulation 2001;103:2828-2833.

57. Jun M, Foote C, Lv J, et al. Effects of fibrates on cardiovascular outcomes: a systematic review and meta-analysis. Lancet 2010;375:1875-1884.

58. Hart RG, Pearce LA, Aguilar MI. Meta-analysis: antithrombotic therapy to prevent stroke in patients who have nonvalvular atrial fibrillation. Ann Intern Med 2007;146:857-867.

59. Andersen KK, Olsen TS. Reduced poststroke mortality in patients with stroke and atrial fibrillation treated with anticoagulants: results from a Danish quality-control registry of 22,179 patients with ischemic stroke. Stroke 2007;38:259-263.

60. Fuster V, Ryden LE, Cannom DS, et al. ACC/AHA/ESC 2006 Guidelines for the Management of Patients with Atrial Fibrillation: a report of the American College of Cardiology/American Heart Association Task Force on Practice Guidelines and the European Society of Cardiology Committee for Practice Guidelines (Writing Committee to Revise the 2001 Guidelines for the Management of Patients With Atrial Fibrillation): developed in collaboration with the European Heart Rhythm Association and the Heart Rhythm Society. Circulation 2006;114:e257-e354.

61. Lip GY, Nieuwlaat R, Pisters R, Lane DA, Crijns HJ. Refining clinical risk stratification for predicting stroke and thromboembolism in atrial fibrillation using a novel risk factor-based approach: the euro heart survey on atrial fibrillation. Chest 2010;137:263-272.

62. Connolly SJ, Ezekowitz MD, Yusuf S, et al. Dabigatran versus warfarin in patients with atrial fibrillation. $\mathrm{N}$ Engl $\mathrm{J}$ Med 2009;361:1139-1151.

63. Mahaffey KW, Fox KAA. ROCKETAF - Rivaroxaban Oncedaily oral direct factor $\mathrm{Xa}$ inhibiation compared with vitamin $\mathrm{K}$ antagnosim for prevention of stroke and embolism Trial in Atrial Fibrillation. American Heart Association Scientific Session, Chicago, Illinois, November 2010.

64. Connolly S, Pogue J, Hart R, et al. Clopidogrel plus aspirin versus oral anticoagulation for atrial fibrillation in the Atrial fibrillation Clopidogrel Trial with Irbesartan for prevention of Vascular Events (ACTIVE W): a randomised controlled trial. Lancet 2006;367:1903-1912.

65. Connolly SJ, Pogue J, Hart RG, et al. Effect of clopidogrel added to aspirin in patients with atrial fibrillation. $\mathrm{N}$ Engl $\mathrm{J}$ Med 2009;360:2066-2078.

66. Barnett SD, Ad N. Surgical ablation as treatment for the elimination of atrial fibrillation: a meta-analysis. J Thorac Cardiovasc Surg 2006;131:1029-1035.

67. Nair GM, Nery PB, Diwakaramenon S, Healey JS, Connolly SJ, Morillo CA. A systematic review of randomized trials comparing radiofrequency ablation with antiarrhythmic medications in patients with atrial fibrillation. J Cardiovasc Electrophysiol 2009;20:138-144.

68. Bonanno C, Paccanaro M, La Vecchia L, Ometto R, Fontanelli A. Efficacy and safety of catheter ablation versus antiarrhythmic drugs for atrial fibrillation: a meta-analysis of randomized trials. J Cardiovasc Med (Hagerstown) 2010;11:408-418.

69. Block PC, Burstein S, Casale PN, et al. Percutaneous left atrial appendage occlusion for patients in atrial fibrillation suboptimal for warfarin therapy: 5-year results of the PLAATO (Percutaneous Left Atrial Appendage Transcatheter Occlusion) Study. JACC Cardiovasc Interv 2009;2:594-600.

70. Holmes DR, Reddy VY, Turi ZG, et al. Percutaneous closure of the left atrial appendage versus warfarin therapy for prevention 
of stroke in patients with atrial fibrillation: a randomised noninferiority trial. Lancet 2009;374:534-542.

71. Shah RS, Cole JW. Smoking and stroke: the more you smoke the more you stroke. Expert Rev Cardiovasc Ther 2010;8:917-932.

72. Boffetta P, Straif K. Use of smokeless tobacco and risk of myocardial infarction and stroke: systematic review with metaanalysis. BMJ 2009;339:b3060.

73. Lawlor DA, Song YM, Sung J, Ebrahim S, Smith GD. The association of smoking and cardiovascular disease in a population with low cholesterol levels: a study of 648,346 men from the Korean National Health System Prospective Cohort Study. Stroke 2008;39:760-767.

74. Kenfield SA, Stampfer MJ, Rosner BA, Colditz GA. Smoking and smoking cessation in relation to mortality in women. JAMA 2008;299:2037-2047.

75. Elkind MS, Sciacca R, Boden-Albala B, Rundek T, Paik MC, Sacco $\mathrm{RL}$. Moderate alcohol consumption reduces risk of ischemic stroke: the Northern Manhattan Study. Stroke 2006;37:13-19.

76. Joosten MM, Beulens JW, Kersten S, Hendriks HF. Moderate alcohol consumption increases insulin sensitivity and ADIPOQ expression in postmenopausal women: a randomised, crossover trial. Diabetologia 2008;51:1375-1381.

77. Patra J, Taylor B, Irving H, et al. Alcohol consumption and the risk of morbidity and mortality for different stroke types - a systematic review and meta-analysis. BMC Public Health 2010;10:258.

78. Chobanian AV, Hill M. National Heart, Lung, and Blood Institute Workshop on Sodium and Blood Pressure : a critical review of current scientific evidence. Hypertension 2000;35:858-863.

79. Sacks FM, Rosner B, Kass EH. Blood pressure in vegetarians. Am J Epidemiol 1974;100:390-398.

80. Appel LJ, Moore TJ, Obarzanek E, et al. A clinical trial of the effects of dietary patterns on blood pressure. DASH Collaborative Research Group. N Engl J Med 1997;336:1117-1124.

81. He FJ, Nowson CA, MacGregor GA. Fruit and vegetable consumption and stroke: meta-analysis of cohort studies. Lancet 2006;367:320-326.

82. Fung TT, Chiuve SE, McCullough ML, Rexrode KM, Logroscino G, Hu FB. Adherence to a DASH-style diet and risk of coronary heart disease and stroke in women. Arch Intern Med 2008;168:713720.

83. Singh RB, Dubnov G, Niaz MA, et al. Effect of an IndoMediterranean diet on progression of coronary artery disease in high risk patients (Indo-Mediterranean Diet Heart Study): a randomised single-blind trial. Lancet 2002;360:1455-1461.

84. de Lorgeril M, Salen P, Martin JL, Monjaud I, Delaye J, Mamelle $\mathrm{N}$. Mediterranean diet, traditional risk factors, and the rate of cardiovascular complications after myocardial infarction: final report of the Lyon Diet Heart Study. Circulation 1999;99:779785.

85. Virtanen JK, Siscovick DS, Longstreth WT Jr., Kuller LH, Mozaffarian D. Fish consumption and risk of subclinical brain abnormalities on MRI in older adults. Neurology 2008;71:439-446.

86. Lee CD, Folsom AR, Blair SN. Physical activity and stroke risk: a meta-analysis. Stroke 2003;34:2475-2481.

87. Wendel-Vos GC, Schuit AJ, Feskens EJ, et al. Physical activity and stroke. A meta-analysis of observational data. Int $\mathbf{J}$ Epidemiol 2004;33:787-798.

88. Patel SC, Levine SR, Tilley BC, et al. Lack of clinical significance of early ischemic changes on computed tomography in acute stroke. JAMA 2001;286:2830-2838.

89. Davidson LE, Hudson R, Kilpatrick K, et al. Effects of exercise modality on insulin resistance and functional limitation in older adults: a randomized controlled trial. Arch Intern Med 2009;169:122-131.

90. Greenlund KJ, Giles WH, Keenan NL, Croft JB, Mensah GA. Physician advice, patient actions, and health-related quality of life in secondary prevention of stroke through diet and exercise. Stroke 2002;33:565-570.

91. Mostofsky E, Laier E, Levitan EB, Rosamond WD, Schlaug G, Mittleman MA. Physical activity and onset of acute ischemic stroke: the stroke onset study. Am J Epidemiol 2011;173:330 336.

92. Whitlock G, Lewington S, Sherliker P, et al. Body-mass index and cause-specific mortality in 900000 adults: collaborative analyses of 57 prospective studies. Lancet 2009;373:1083-1096.

93. Strazzullo P, D'Elia L, Cairella G, Garbagnati F, Cappuccio FP, Scalfi L. Excess body weight and incidence of stroke: metaanalysis of prospective studies with 2 million participants. Stroke;41:e418-e426.

94. Vemmos K, Ntaios G, Spengos K, et al. Association between obesity and mortality after acute first-ever stroke: the obesitystroke paradox. Stroke;42:30-36.

95. Kim BJ, Lee SH, Ryu WS, Kim CK, Lee J, Yoon BW. Paradoxical longevity in obese patients with intracerebral hemorrhage. Neurology;76:567-573.

96. Towfighi A, Ovbiagele B. The impact of body mass index on mortality after stroke. Stroke 2009;40:2704-2708.

97. Goldstein LB, Adams R, Alberts MJ, et al. Primary prevention of ischemic stroke: a guideline from the American Heart Association/American Stroke Association Stroke Council: cosponsored by the Atherosclerotic Peripheral Vascular Disease Interdisciplinary Working Group; Cardiovascular Nursing Council; Clinical Cardiology Council; Nutrition, Physical Activity, and Metabolism Council; and the Quality of Care and Outcomes Research Interdisciplinary Working Group: the American Academy of Neurology affirms the value of this guideline. Stroke 2006;37:1583-1633.

98. Kurl S, Laukkanen JA, Niskanen L, et al. Metabolic syndrome and the risk of stroke in middle-aged men. Stroke 2006;37:806-811.

99. Bassetti C, Aldrich MS, Chervin RD, Quint D. Sleep apnea in patients with transient ischemic attack and stroke: a prospective study of 59 patients. Neurology 1996;47:1167-1173.

100. Bravata DM, Concato J, Fried T, et al. Auto-titrating continuous positive airway pressure for patients with acute transient ischemic attack: a randomized feasibility trial. Stroke 2010;41:1464-1470.

101. Martinez-Garcia MA, Galiano-Blancart R, Roman-Sanchez P, Soler-Cataluna JJ, Cabero-Salt L, Salcedo-Maiques E. Continuous positive airway pressure treatment in sleep apnea prevents new vascular events after ischemic stroke. Chest 2005;128:2123-2129.

102. Martinez-Garcia MA, Soler-Cataluna JJ, Ejarque-Martinez L, et al. Continuous positive airway pressure treatment reduces mortality in patients with ischemic stroke and obstructive sleep apnea: a 5-year follow-up study. Am J Respir Crit Care Med 2009; 180:36-41.

103. Kanagala R, Murali NS, Friedman PA, et al. Obstructive sleep apnea and the recurrence of atrial fibrillation. Circulation 2003;107:2589-2594.

104. Danesh J, Collins R, Peto R. Lipoprotein(a) and coronary heart disease. Meta-analysis of prospective studies. Circulation 2000;102:1082-1085.

105. Erqou S, Kaptoge S, Perry PL, et al. Lipoprotein(a) concentration and the risk of coronary heart disease, stroke, and nonvascular mortality. JAMA 2009;302:412-423.

106. Kim BS, Jung HS, Bang OY, Chung CS, Lee KH, Kim GM. Elevated serum lipoprotein(a) as a potential predictor for combined intracranial and extracranial artery stenosis in patients with ischemic stroke. Atherosclerosis 2010;212:682-688.

107. Elam MB, Hunninghake DB, Davis KB, et al. Effect of niacin on lipid and lipoprotein levels and glycemic control in patients with diabetes and peripheral arterial disease: the ADMIT study: a randomized trial. Arterial Disease Multiple Intervention Trial. JAMA 2000;284:1263-1270. 
108. Goldberg A, Alagona P Jr., Capuzzi DM, et al. Multiple-dose efficacy and safety of an extended-release form of niacin in the management of hyperlipidemia. Am J Cardiol 2000;85:1100-1105.

109. Bruckert E, Labreuche J, Amarenco P. Meta-analysis of the effect of nicotinic acid alone or in combination on cardiovascular events and atherosclerosis. Atherosclerosis 2010;210:353-361.

110. Ridker PM, Manson JE, Buring JE, Shih J, Matias M, Hennekens $\mathrm{CH}$. Homocysteine and risk of cardiovascular disease among postmenopausal women. JAMA 1999;281:1817-1821.

111. Wald DS, Law M, Morris JK. Homocysteine and cardiovascular disease: evidence on causality from a meta-analysis. BMJ 2002;325:1202.

112. Den Heijer M, Lewington S, Clarke R. Homocysteine, MTHFR and risk of venous thrombosis: a meta-analysis of published epidemiological studies. J Thromb Haemost 2005;3:292-299.

113. Durga J, Verhoef P, Bots ML, Schouten E. Homocysteine and carotid intima-media thickness: a critical appraisal of the evidence. Atherosclerosis 2004;176:1-19.

114. Jacques PF, Selhub J, Bostom AG, Wilson PW, Rosenberg IH. The effect of folic acid fortification on plasma folate and total homocysteine concentrations. N Engl J Med 1999;340:1449-1454.

115. Lee M, Hong KS, Chang SC, Saver JL. Efficacy of homocysteine-lowering therapy with folic Acid in stroke prevention: a meta-analysis. Stroke 2010;41:1205-1212.

116. Morris JG, Singh S, Fisher M. Testing for inherited thrombophilias in arterial stroke: can it cause more harm than good? Stroke 2010;41:2985-2990.

117. Hamedani AG, Cole JW, Mitchell BD, Kittner SJ. Meta-analysis of factor $\mathrm{V}$ Leiden and ischemic stroke in young adults: the importance of case ascertainment. Stroke 2010;41:1599-1603.

118. Weber R, Goertler M, Benemann J, Diener HC, Weimar C. Prognosis after cryptogenic cerebral ischemia in patients with coagulopathies. Cerebrovasc Dis 2009;28:611-617.

119. Kenet G, Lutkhoff LK, Albisetti M, et al. Impact of thrombophilia on risk of arterial ischemic stroke or cerebral sinovenous thrombosis in neonates and children: a systematic review and meta-analysis of observational studies. Circulation 2010;121:1838-1847.

120. Miyakis S, Lockshin MD, Atsumi $\mathrm{T}$, et al. International consensus statement on an update of the classification criteria for definite antiphospholipid syndrome (APS). J Thromb Haemost 2006;4:295-306.

121. The Antiphospholipid Antibodies in Stroke 1206 Study (APASS) Group. Anticardiolipin antibodies are an independent risk factor for first ischemic stroke. Neurology 1993;43:2069-2073.

122. Ginsburg KS, Liang MH, Newcomer L, et al. Anticardiolipin antibodies and the risk for ischemic stroke and venous thrombosis. Ann Intern Med 1992;117:997-1002.

123. Janardhan V, Wolf PA, Kase CS, et al. Anticardiolipin antibodies and risk of ischemic stroke and transient ischemic attack: the Framingham cohort and offspring study. Stroke 2004;35:736741.

124. Tuhrim S, Rand $\mathrm{JH}, \mathrm{Wu} \mathrm{XX}$, et al. Elevated anticardiolipin antibody titer is a stroke risk factor in a multiethnic population independent of isotype or degree of positivity. Stroke 1999;30:1561-1565.

125. Ahmed E, Stegmayr B, Trifunovic J, Weinehall L, Hallmans G, Lefvert AK. Anticardiolipin antibodies are not an independent risk factor for stroke: an incident case-referent study nested within the MONICA and Vasterbotten cohort project. Stroke 2000;31:1289-1293.

126. Levine SR, Brey RL, Tilley BC, et al. Antiphospholipid antibodies and subsequent thrombo-occlusive events in patients with ischemic stroke. JAMA 2004;291:576-584.

127. Urbanus RT, Siegerink B, Roest M, Rosendaal FR, de Groot PG, Algra A. Antiphospholipid antibodies and risk of myocardial infarction and ischaemic stroke in young women in the RATIO study: a case-control study. Lancet Neurol 2009;8:998-1005.

128. Ruiz-Irastorza G, Crowther M, Branch W, Khamashta MA. Antiphospholipid syndrome. Lancet 2010;376:1498-1509. 